\title{
Investigação do fluxo expiratório máximo em gestantes saudáveis
}

\author{
Peak expiratory flow analysis in healthy pregnant women \\ Gustavo Antonio Neppelenbroek ${ }^{1}$, Francisco Mauad-Filho², Sérgio Pereira da Cunha ${ }^{3}$, \\ Geraldo Duarte ${ }^{4}$, Antonio Gadelha Costa ${ }^{5}$, Patrícia Spara ${ }^{6}$, Glauce Maria Gelonezi
}

\section{RESUMO}

Objetivo: investigar os valores normais de fluxo expiratório máximo para gestantes normais, com uso de aparelho expiratório portátil (Mini-Wright Peak Flow Meter), relacionando as medidas obtidas com estatura, índice de massa corporal (IMC) e idade de cada paciente, ao longo da gestação. Métodos: estudo prospectivo longitudinal com 26 grávidas acompanhadas do primeiro trimestre até 36 semanas, sendo examinadas a cada quatro semanas. Durante sete visitas, as gestantes exalaram forçadamente três vezes no aparelho respiratório portátil, sendo considerado o maior valor como fluxo expiratório máximo. Todas as medidas foram realizadas de forma assistida, pelo mesmo pesquisador. O coeficiente de Pearson foi utilizado para calcular as correlações entre fluxo e IMC, entre fluxo e estatura e entre fluxo e idade da paciente. Resultados: a variação do fluxo na gestação pode ser determinada por fluxo $=328,32-0,07$ x semana, com coeficiente de Pearson igual a zero. Para verificar se houve diferença entre os coeficientes de correlação entre IMC e fluxo, em cada intervalo estudado, comparouse o menor coeficiente $(0,47$ da semana 30 , fluxo $=123,49+7,64 \times$ IMC $)$ com o maior $(0,59$ da semana 34 , fluxo $=87,77+9,05$ $\mathrm{x}$ IMC) e foi obtido o valor de 0,22 , entre as variáveis fluxo e IMC. Entre estatura e fluxo, houve correlação positiva (Pearson $=0,61$, com fluxo $=-477,47+497,38 \times$ estatura. $O$ coeficiente de correlação entre fluxo e idade foi de 0,24 , não se obtendo equação linear neste caso. Conclusões: os valores de fluxo expiratório máximo não se modificaram ao longo da gestação. Fluxos mais elevados foram encontrados em pacientes mais altas. Gestantes com maiores IMC prévios à gestação apresentaram maiores fluxos. Não houve correlação entre fluxo e idade materna.

PALAVRAS-CHAVE: Fluxo expiratório máximo; Índice de massa corporal; Gravidez normal

\section{ABSTRACT}

Purpose: to investigate the normal peak expiratory flow values in healthy pregnant women employing a portable expiratory apparatus (Mini-Wright Peak Flow Meter), and to relate the obtained measurements to each patient's height, body mass index (BMI) and age, along gestation. Methods: a longitudinal prospective study including 26 pregnant women followed up from the first trimester to the 36th week of gestation and examined every four weeks. On the occasion of seven visits, the pregnant women performed forced exhaling into a portable expiratory apparatus three times, with the highest value being considered the peak expiratory flow. All measurements were made under the same investigator's supervision in order to reduce the margin of error. Pearson coefficient was used to calculate the correlation between flow and BMI, between flow and patient's height, and between flow and patient's age. Results: the variation in flow values during pregnancy can be determined by flow $=328.32-0.07 \mathrm{x}$ week, with a Pearson coefficient equal to zero. To determine whether there was a difference in the correlation coefficients between BMI and flow, we compared the lowest coefficient ( 0.47 for week 30 , flow $=123.49+7.64 \times \mathrm{BMI})$ with the highest coefficient $(0.59$ for week 34 , flow $=87.77+9.05 \times \mathrm{BMI})$ of each studied time interval and obtained a value of 0.22 , indicating a good correlation between the flow and BMI variables. There was a positive correlation between height and flow (Pearson $=0.61$ ), with flow $=-477.47+497.38 \mathrm{x}$ height. The correlation coefficient between flow and age was 0.24, with the equation obtained in this case being non-linear. Conclusions: peak expiratory flow values did not change along gestation. Higher flows were observed in taller women. Pregnant women with a higher BMI before gestation presented higher flows. There was no correlation between flow and maternal age.

KEYWORDS: Peak expiratory flow; Body mass index; Normal pregnancy

Departamento de Ginecologia e Obstetrícia da Faculdade de Medicina de Ribeirão Preto - Universidade de São Paulo

1,5,6,7 Pós-Graduando do Departamento de Ginecologia e Obstetrícia da Faculdade de Medicina de Ribeirão Preto - Universidade de São Paulo

2,3,4 Docente do Departamento de Ginecologia e Obstetricia da Faculdade de Medicina de Ribeirão Preto - Universidade de São Paulo

Correspondência: Francisco Mauad-Filho

Hospital das Clínicas da Faculdade de Medicina de Ribeirão Preto - USP

Avenida Bandeirantes, 3900 - 14049-900 - Ribeirão Preto - SP - Telefone: (16) 633-0216 - e-mail: mauad@ultra-sonografia.com.br

Recebido em: 20/7/2004 Aceito com modificações em: 10/1/2005 
Introdução

A espirometria de fluxo é o teste-padrão para avaliar a função pulmonar e suas alterações, inclusive durante a gravide $z^{1,2}$. A gravidez imprime modificações importantes na fisiologia respiratória da mulher, com aumento de $20 \%$ no consumo de oxigênio e aumento de $15 \%$ na taxa de metabolismo materno ${ }^{3}$. Esta demanda extra é alcançada devido a acréscimo de 40 a $50 \%$ no volume minuto respiratório, de $7,5 \mathrm{~L} / \mathrm{min}$ (litros/minuto) para $10,5 \mathrm{~L} / \mathrm{min}$, resultado mais do aumento do volume corrente do que de alterações na freqüência respiratória. Isso implica aumento da profundidade da respiração, ocorrendo aumento de $50 \%$ da ventilação pulmonar, com manutenção da freqüência respiratória ${ }^{3}$.

A permeabilidade das vias aéreas é aumentada e a resistência pulmonar é reduzida, possivelmente pela ação da progesterona ${ }^{3}$. Além disso, esse hormônio da gravidez estimula o centro respiratório ${ }^{3}$. Esta hiperventilação causa aumento da pressão parcial de oxigênio e redução da pressão parcial de gás carbônico, com queda compensatória no bicarbonato sérico de $18-22 \mathrm{mmol} / \mathrm{L}$. Portanto respiração compativel com padrão de alcalose é esperada na grávida ${ }^{4}$, parcialmente compensada por acidose metabólica moderada ${ }^{5}$. Diante das mudanças acima descritas, ocorre diminuição da capacidade residual funcional na gestação ${ }^{4,6}$. As inúmeras adaptações no trato respiratório durante a gravidez são significativas ${ }^{3}$. Em fases avançadas no período gestacional, a função pulmonar pode ser gravemente afetada por doenças pulmonares agudas e crônicas ${ }^{7}$, sobretudo a asma.

As alterações da fisiologia pulmonar durante a gravidez podem ser mensuradas por técnicas espirométricas diretas ${ }^{2}$. Essas mudanças podem ser sumarizadas pelas seguintes alterações: aumento da capacidade vital (de 100 a $200 \mathrm{~mL}$ ), aumento da capacidade inspiratória (de $300 \mathrm{~mL}$ em fases tardias), redução do volume de reserva expiratório (de 1.300 a $1.100 \mathrm{~mL}$ ), redução da capacidade funcional (para cerca de $500 \mathrm{~mL}$ ), aumento do volume corrente (de $500 \mathrm{ml}$ para $700 \mathrm{~mL}$ ) e manutenção do volume expiratório forçado em um segundo ${ }^{3}$. O fluxo expiratório máximo é parâmetro prático, resumido e reprodutível para medir a função pulmonar, medido isoladamente com aparelho portátil ${ }^{8}$. O National Institutes of Health ${ }^{9}$ e o American College of Obstetricians and Gynecologists ${ }^{10}$ preconizam a utilização do fluxo expiratório máximo como modalidade para diagnóstico e de seguimento de grávidas com asma.

Tendo em vista a escassez de trabalhos na literatura internacional e a falta de estudos na literatura nacional sobre a curva normal de fluxo expiratório máximo em grávidas normais, ressalta-se a importância da realização deste estudo.

O objetivo do presente estudo é investigar os valores normais de fluxo expiratório máximo para gestantes, em etapas do periodo gestacional, relacionando as medidas obtidas de fluxo expiratório máximo com o índice de massa corporal (IMC), a idade materna e a estatura materna.

\section{Métodos}

Foi realizado estudo prospectivo longitudinal, no qual 26 gestantes consideradas clinicamente normais foram avaliadas por medidor portátil para aferição de fluxo expiratório máximo. As pacientes eram oriundas do Ambulatório de Pré-Natal do Departamento de Ginecologia e Obstetrícia da Faculdade de Medicina de Ribeirão Preto da Universidade de São Paulo.

As pacientes foram selecionadas conforme os critérios de inclusão e exclusão. Os critérios de inclusão foram: gestação única, pacientes com idade gestacional inicial de até 16 semanas, com o tempo de gestação bem estabelecido (baseada na data da última menstruação e confirmado por exame ultra-sonográfico realizado até a $12^{\mathrm{a}}$ semana ou por dois exames ultra-sonográficos concordantes realizados entre a $12^{\mathrm{a}}$ e a $20^{\mathrm{a}}$ semana de gestação). Todas as participantes teriam que aceitar a realização de medidas mensais do fluxo expiratório máximo, durante os retornos habituais do pré-natal. Foram excluídas grávidas com doenças maternas associadas ou intercorrentes à gestação estudada, com malformação fetal e exposição inalatória (informação obtida por meio de anamnese) de substâncias tóxicas por vício ou risco ocupacional (fumo, maconha, etc.). Todas as pacientes assinaram o Termo de Consentimento Informado. O estudo na forma em que se apresenta foi aprovado pelo Comitê de Ética em Pesquisa do Hospital das Clínicas da Faculdade de Medicina de Ribeirão Preto da Universidade de São Paulo, conforme o Processo HCRP n ${ }^{\circ}$. 6432/ 2002.

A idade e a estatura de cada gestante foram avaliadas no momento da primeira consulta. As evoluções do peso e do IMC maternas foram medidas mensalmente. Os valores foram anotados em ficha clínica da paciente e posteriormente submetidos a uma análise descritiva.

Em cada uma das sete aferições, com periodicidade mensal, foram realizadas medidas do fluxo expiratório máximo. As possiveis queixas rele- 
vantes no decorrer da gestação foram anotadas. Dessa forma, a manutenção dos critérios de inclusão e exclusão foi assegurada.

As medidas do fluxo expiratório máximo com aparelho Mini-Wright Peak Flow Meter foram efetuadas, sob supervisão de observador único, obedecendo aos seguintes critérios: explicação para a paciente sobre a forma correta de segurar o medidor de maneira que os dedos não cubram a escala medidora ou impeçam o movimento do cursor, em posição ortostática, solicitação para a paciente respirar profundamente, levando o medidor horizontalmente para a boca, fechando os lábios em volta do medidor e assoprando com força (como a ação de apagar uma vela), anotação do valor indicado pela agulha na escala, voltando a colocar a agulha indicadora no zero e solicitando para o paciente repetir o procedimento por duas vezes, conseguindo um total de três leituras. O maior valor obtido foi anotado.

As variáveis analisadas foram os valores de fluxo expiratório máximo (L/ $\mathrm{min}$ ) ao longo da gestação, correlacionando-os com o IMC $\left(\mathrm{kg} / \mathrm{m}^{2}\right)$, a estatura $(\mathrm{m})$ e a idade materna (anos). Os dados obtidos foram tabulados no programa Microsoft Excel. Para a análise estatística dos resultados foram utilizados a análise de regressão linear e o coeficiente de correlação de Pearson. Para significância estatística considerou-se $\mathrm{p}<0,05$.

Todas as medidas foram realizadas em medidores de fluxo individuais, o Mini-Wright Peak Flow Meter. É fabricado pela empresa inglesa Clement Clarke International. É disponivel nas versões infantil e adulta, sendo esta última a utilizada neste experimento. O fluxo expiratório máximo é aferido por uma escala padronizada pela American Thorax Society ${ }^{11}$, de 60 a 880 L/min, de calibração manual.

Para calcular o tamanho da amostra (N), foi estabelecido erro $\alpha$ de $5 \%$, com intervalo de confiança de $95 \%$ e erro $\beta$ de $20 \%$. Foi utilizada fórmula ${ }^{12}\left(\mathrm{~N}=Z^{2} \times(\mathrm{p} \times \mathrm{q}) / \mathrm{i}^{2}\right)$ onde $Z$ significa $\mathrm{o}$ valor de nível de significância desejado, p significa a proporção estimada para a ocorrência do evento em estudo, q significa o complementar de $\mathrm{p}$ e i significa a variabilidade aceitável da referida proporção.

Foi estabelecido nível de significância de 5\%, determinando um $Z$ de 1,96. Em nosso meio, não há literatura sobre os valores de fluxos expiratórios máximos em gestantes. Dessa forma, em que pese o caráter inédito da proposição do presente estudo na literatura nacional, procurou-se estudar a variação do fluxo expiratório máximo como em gestantes americanas ${ }^{13}$. Não houve variação significativa do fluxo expiratório máximo $(\mathrm{p}=95 \%)$ ao lon- go da gestação nesse estudo. Portanto, adotou-se $95 \%(0,95)$ como valor aproximado de $\mathrm{p}$, o que determinou valor de q de $5 \%(0,05)$. Finalmente, foi determinado que seria aceito valor de i de até $10 \%(0,1)$.

Dessa forma, tem-se: $N=1,96 \times(0,95 \times$ $0,05) / 0,1^{2}$ e $\mathrm{N}=3,84 \times 0,0475 / 0,01$, obtendo-se $\mathrm{N}$ $=18,24$.

Ao valor aproximado de 19 pacientes foram acrescentados $20 \%$ no intuito de prevenir possíveis perdas durante a coleta de dados e seguimento das gestantes, o que determinou uma meta amostral de 22 grávidas. Foi possivel a conclusão do estudo com a observação de 26 gestantes, do primeiro trimestre à resolução da gestação.

\section{Resultados}

A idade materna variou de 16 a 36 anos, com média de 23,2 anos. O peso pré-gestacional aferido nas voluntárias variou de 40,0 a $94,0 \mathrm{~kg}$, com média de $60,5 \mathrm{~kg}$. O peso final constatado variou de 50,0 a $98,0 \mathrm{~kg}$, com média de $71,8 \mathrm{~kg}$. A estatura aferida à avaliação da seleção variou de 1,45 a $1,74 \mathrm{~m}$, com média de $1,60 \mathrm{~m}$. Outro parâmetro importante avaliado foi o IMC, sendo que ocorreu variação entre 18,7 e $34,8 \mathrm{~kg} / \mathrm{m}^{2}$, com média de $25,2 \mathrm{~kg} / \mathrm{m}^{2}$ (Tabela 1 ).

Tabela 1 - Distribuição da casuística quanto às características clínicas e antropométricas.

\begin{tabular}{lcc}
\hline & Média \pm desvio padrão & Mediana \\
\hline Idade (anos) & $23,2 \pm 5,0$ & 22,0 \\
Peso pré-gestacional $(\mathrm{kg})$ & $60,5 \pm 13,8$ & 55,3 \\
Peso final $(\mathrm{kg})$ & $71,8 \pm 14,7$ & 70,0 \\
Estatura $(\mathrm{m})$ & $1,6 \pm 0,07$ & 1,6 \\
IMC $\left(\mathrm{kg} / \mathrm{m}^{2}\right)$ & $25,2 \pm 3,8$ & 25,3 \\
\hline
\end{tabular}

IMC = índice de massa corporal.

Mensalmente, os valores de fluxo expiratório máximo das 26 gestantes foram mensurados, sendo calculados média, desvio padrão e mediana para cada intervalo estudado (Tabela 2).

Para verificar se existe alguma correlação entre as variáveis fluxo e as semanas de gestação, realizou-se análise de regressão linear. O fluxo expiratório máximo foi considerado como variável dependente e cada intervalo das semanas de gestação como variável preditora. Equação de primeiro grau em que o fluxo expiratório máximo permanece com valores estáveis ao longo da ges- 
tação foi obtida: fluxo $=328.32-0.07 \times$ semana, com $\mathrm{p}=0,90$ (Figura 1).

Tabela 2 - Média, mediana e desvio padrão dos fluxos para cada intervalo de 4 semanas estudado.

\begin{tabular}{lcc}
\hline $\begin{array}{l}\text { Intervalo de } \\
\text { semanas }\end{array}$ & $\begin{array}{c}\text { Média } \pm \\
\text { desvio padrão }(\mathrm{L} / \mathrm{min})\end{array}$ & $\begin{array}{c}\text { Mediana } \\
(\mathrm{L} / \mathrm{min})\end{array}$ \\
\hline $12-16$ & $323,4 \pm 66,9$ & 330,0 \\
$16-20$ & $332,3 \pm 56,2$ & 340,0 \\
$20-24$ & $330,7 \pm 62,0$ & 350,0 \\
$24-28$ & $320,0 \pm 59,5$ & 320,0 \\
$28-32$ & $325,7 \pm 57,0$ & 340,0 \\
$32-36$ & $326,9 \pm 55,8$ & 340,0 \\
$36-40$ & $326,1 \pm 59,6$ & 340,0 \\
\hline
\end{tabular}

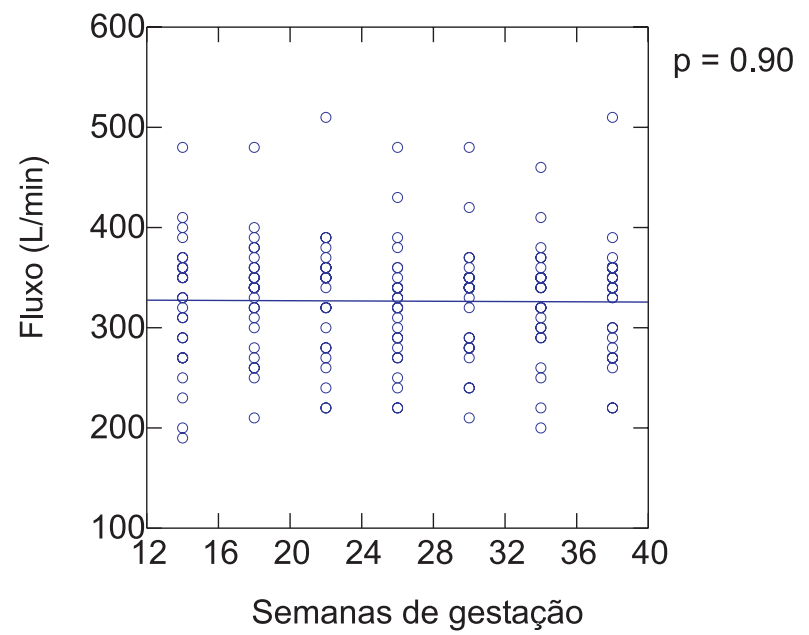

Figura 1 - Relação entre fluxo expiratório máximo e idade gestacional.

Relacionamos o IMC com o fluxo expiratório máximo, para cada intervalo de 4 semanas estudado para as 26 gestantes. Para cada intervalo, foi calculado o coeficiente de Pearson e a equação de regressão linear com seus respectivos $\mathrm{p}$ (significância) (Tabela 3).

Para verificar se houve diferença entre os coeficientes de correlação entre IMC e fluxo, comparou-se o menor coeficiente $(0,47$ da semana 30) com o maior $(0,59$ da semana 34$)$ e foi obtido o valor de 0,22 . Como as amostras são todas do mesmo tamanho $(n=26)$, não houve diferença significativa $(0,22)$ entre a diferença entre os coeficientes de correlação de cada semana. Esse resultado indica correlação positiva entre as variáveis fluxo e IMC, isto é, quanto maior o valor de IMC apresentado, maior o fluxo expiratório máximo da paciente.
Tabela 3 - Coeficiente de Pearson e equação de regressão do valor de fluxo para cada intervalo de 4 semanas estudado, de acordo com o índice de massa corporal (IMC) de cada gestante.

\begin{tabular}{lrlc}
\hline $\begin{array}{l}\text { Intervalo de } \\
\text { semanas }\end{array}$ & Equação do fluxo & $\mathbf{p}$ & $\begin{array}{c}\text { Coeficiente } \\
\text { de Pearson }\end{array}$ \\
\hline $12-16$ & $118,90+8,84 \times$ IMC & 0,01 & 0,48 \\
$16-20$ & $153,96+7,52 \times$ IMC & 0,01 & 0,48 \\
$20-24$ & $96,34+9,57 \times$ IMC & 0,006 & 0,53 \\
$24-28$ & $83,74+9,38 \times$ IMC & 0,005 & 0,53 \\
$28-32$ & $123,49+7,64 \times$ IMC & 0,02 & 0,47 \\
$32-36$ & $83,77+9,05 \times$ IMC & 0,001 & 0,59 \\
$36-40$ & $103,76+8,09 \times$ IMC & 0,005 & 0,54 \\
\hline
\end{tabular}

Como a variação de fluxo não é significativa ao longo das semanas (Figura 1), a média aritmética dos fluxos expiratórios máximos de cada paciente foi comparada com sua respectiva estatura.

Para verificar se existia alguma correlação entre as variáveis estatura materna e as semanas de gestação, realizou-se cálculo do coeficiente de correlação de Pearson e análise por regressão linear. O coeficiente de correlação linear de Pearson entre fluxo médio e estatura foi de 0,61 , com $\mathrm{p}=0,001$; portanto, houve correlação positiva bastante significativa entre as variáveis. A reta estimada para essa relação pode ser estabelecida por fluxo médio $=-477,47+497.38$ $\mathrm{x}$ estatura, com $\mathrm{p}=0,001$, demonstrando a significância do ajuste, $\mathrm{R}^{2}=36,8 \%$. O percentual de variação do fluxo médio é explicado pela variação da estatura (Figura 2).

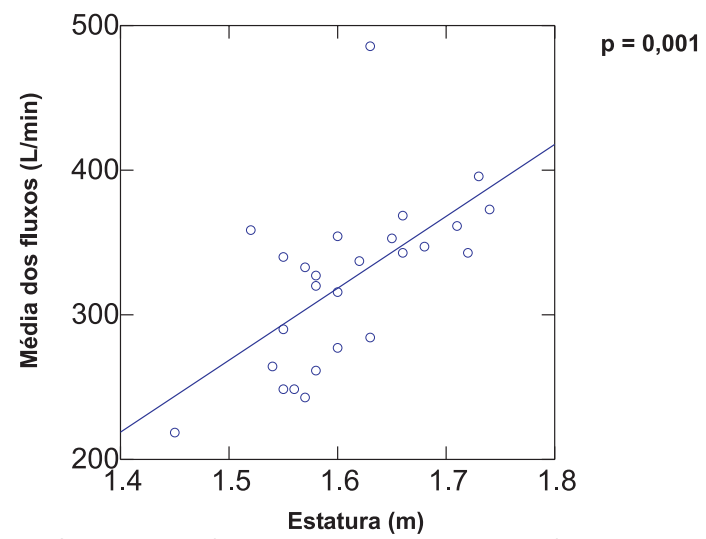

Figura 2 - Correlação significativa e positiva entre valores de fluxo médio e estatura.

Como a variação de fluxo não foi significativa ao longo das semanas (Figura 2), a média aritmética dos fluxos expiratórios máximos de 
cada paciente foi comparada com sua respectiva idade. Para a variável idade, nenhum modelo testado apresentou resultado significativo, portanto não se realizou ajuste, não havendo relação entre as variáveis. O coeficiente de correlação linear de Pearson entre fluxo médio e idade foi de 0,24 , com $p=0,23$, portanto houve correlação positiva fraca entre as variáveis, porém não significativa (Figura 3).

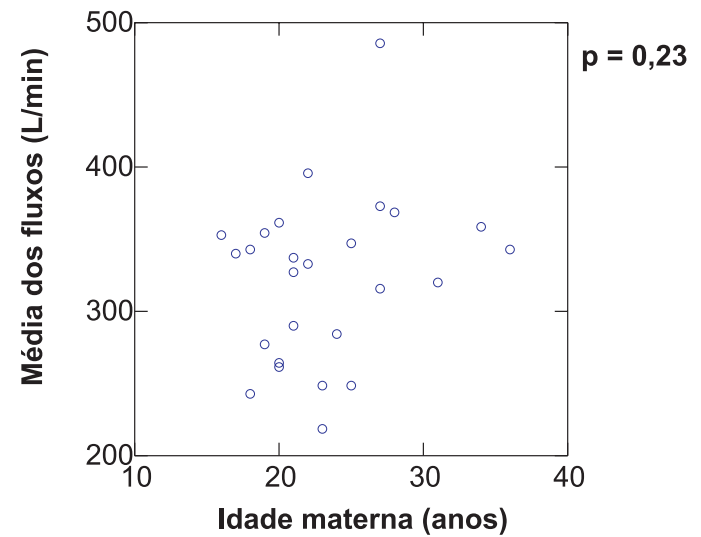

Figura 3 - Relação entre médias dos fluxos expiratórios máximos e idade materna.

\section{Discussão}

Com relação à mensuração da função pulmonar, a espirometria é considerada o melhor método ${ }^{14}$. No entanto, o espirômetro de água (mais difundido) tem dimensões consideráveis, demandando técnicos especializados, com tempo adequado para o procedimento, em horários determinados, exigindo que a paciente sempre se desloque para avaliação. Como vantagem, é método simples para estudar a ventilação pulmonar, permitindo registrar o volume de ar que se movimenta durante a expiração e a inspiração ${ }^{14}$. Dessa forma, podem ser estudados os volumes corrente, de reserva inspiratório e de reserva expiratório ${ }^{15}$.

O fluxo expiratório corresponde à quantidade de ar expelida em milissegundos, medida em litros por minuto, durante uma expiração forçada. Somente é avaliado em aparelhos portáteis. O espirômetro de água é incapaz de medi-lo por não ter a freqüência de resposta necessária para capturar o evento, além de ter maior resistência interna para suportar altos valores de fluxo expiratório ${ }^{13}$.

A avaliação do fluxo expiratório máximo (aparelho portátil) permite que sejam obtidos valores próximos aos do volume expiratório forçado em um segundo (espirômetro), com correspondência de $98 \%$, entre os dois aparelhos ${ }^{16}$.

O Mini-Wright Peak Flow Meter é aparelho portátil que permite detectar situações subclínicas de doenças respiratórias antes mesmo de sua descompensação ${ }^{13}$, além de importante método de seguimento domiciliar. É de fácil manuseio e aplicabilidade, sendo descartável, leve (73 g) e de uso individual, utilizado rotineiramente pelo próprio paciente.

Como desvantagem, os resultados dependem dos esforços máximos do indivíduo para que os testes sejam válidos, uma vez que a ausência do médico ou do técnico na obtenção dos valores pode criar medidas menores do que as reais ${ }^{13}$. Esse tipo de erro foi evitado no nosso trabalho, uma vez que todas as medidas foram realizadas sob supervisão médica.

No presente estudo, foi mostrado que o fluxo expiratório máximo não se altera com a evolução da gravidez, apesar do ganho de peso durante esse período. Dessa forma, a comparação dos resultados obtidos com os valores de fluxo expiratório máximo padronizado para pacientes não grávidas pode ser realizada. Outros estudos da literatura médica internacional confirmam que o fluxo expiratório máximo não se altera com a evolução da gestação ${ }^{10,13,17,18}$.

A maior parte dos trabalhos sobre função pulmonar em gestantes utilizou-se de espirômetros, descrevendo vários parâmetros da fisiologia pulmonar ${ }^{2,6,19}$, mas, não foi medido o fluxo expiratório máximo, uma vez que somente pode ser avaliado pelo medidor portátil. Em estudo longitudinal em gestantes indianas, em que foi medido o fluxo expiratório máximo por meio do medidor portátil ${ }^{20}$, não ocorreu qualquer correlação entre fluxo expiratório e a altura e a idade. Nesse estudo, os autores demonstraram que o fluxo diminui ao longo da gestação. Salientaram também que os valores absolutos obtidos são menores do que os esperados porque as pacientes indianas apresentam menor volume torácico. Partindo dessa premissa de variação étnica, reforça-se a necessidade da realização do nosso trabalho para aplicação na população brasileira. Inclusive, a Sociedade Americana do Tórax ${ }^{11}$, em revisão de suas normas, recomenda que para cada grupamento populacional sejam escolhidas equações próprias que mais se adaptem à sua realidade.

Os IMCs das gestantes foram avaliados em periodos da idade gestacional (a cada quatro semanas), comparando com os valores de fluxo expiratório máximo. Observamos que os maiores IMCs correspondiam aos maiores valores de fluxo, em todos os intervalos de semanas estudados. 
As pacientes que já apresentavam IMCs mais elevados em etapas iniciais da gestação mantiveramse com os maiores fluxos durante toda a gestação. Portanto, o IMC prévio à gestação foi considerado fator importante nos valores dos fluxos obtidos ao longo da gestação. Este é um achado importante, considerando que o aumento de IMC, causado pelo ganho ponderal que ocorre durante a gravidez, é fator-chave na prevalência da asma ${ }^{21}$. No entanto, a relação positiva entre IMC e asma não é explicada pela alteração do fluxo expiratório máximo, uma vez que este parâmetro manteve-se constante ao longo da gestação no presente estudo. Outros autores ${ }^{22}$ também comprovaram que a variação individual de IMC com perda ou ganho de peso não altera o fluxo expiratório máximo.

Em importante estudo ${ }^{23}$, utilizando espirometria, foi estabelecida correlação positiva entre o volume expiratório forçado em um segundo e a estatura de pacientes não gestantes. Em outro trabalho realizado apenas com gestantes ${ }^{13}$, com uso do aparelho portátil, a existência dessa correlação também foi demonstrada. Esses resultados foram ratificados no nosso trabalho, ou seja, a estatura materna apresenta correlação positiva com o fluxo expiratório máximo.

Em relação à idade materna apresentada, o estudo de referência para a população brasilei$\mathrm{ra}^{23}$ estabelece correlação negativa entre idade e volume expiratório forçado em um segundo, em indivíduos adultos não gestantes. Essa conclusão de que quanto mais idosa a paciente, menor o fluxo, é confirmada em gestantes americanas ${ }^{13}$. Em nosso estudo, entretanto, a idade materna não mostrou ser importante na correção dos valores, sendo que os resultados obtidos não sofreram influência com a variação da idade. A nossa amostra constitui-se de pessoas jovens, com pouca diferença de idade, o que poderia explicar nossos resultados.

O entendimento do comportamento expiratório imprimido pelas modificações corpóreas que a gravidez causa deve ser cada vez mais elucidado. Cada conquista de informação desta cadeia que envolve a respiração, principalmente na gestação, deve ser salientada. O presente trabalho permite criar novos projetos de pesquisa, envolvendo grávidas com doenças pulmonares, sobretudo em casos de asma, pois essa doença respiratória, ao diminuir o fluxo respiratório máximo, dificulta o processo respiratório, trazendo repercussões para o desenvolvimento fetal $^{24}$. Destaca-se a elevada incidência de complicações perinatais, o que já foi constatado por vários autores que estudaram a associação de asma e gravide $z^{25,26}$.
Diante do exposto, o desenvolvimento de uma curva de valores normais para os fluxos expiratórios máximos, em gestantes clinicamente consideradas normais, estabelecida pelo nosso trabalho, contribui para os estudos de fisiologia de respiração durante a gestação.

\section{Referências}

1. Taylor AE, Rehder K, Hyatt RE, Parker JC. Clinical respiratory physiology. $4^{\text {th }}$ ed. Philadelphia: W.B. Saunders; 1989. p. 38-99.

2. Puranik BM, Kaore SB, Kurhade GA, Agrawal SD, Patwardhan SA, Kher JR. A longitudinal study of pulmonary function tests during pregnancy. Indian J Physiol Pharmacol. 1994; 38:129-32.

3. De Swiet M. The respiratory system. In: Hytten FE, Chamberlain G, editors. Clinical Physiology in Obstetrics. $2^{\text {nd }}$ ed. Oxford: Blackwell; 1991. p. 83-7.

4. Stenius-Aarniala B. Pulmonary function during pregnancy in heath and in asthma. In: Schatz M, Zeiger RS, editors. Asthma and Allergy in Pregnancy and Early Infancy. New York: Marcel Decker; 1993. p. 53-62.

5. Mauad-Filho F, Nunes de Moraes E, Vaz Parente J, Gomes UA, Leite de Carvalho R. Effect of glucose infusion on the maternal and fetal acid-base equilibrium during labor. J Perinat Med. 1982; 10:99-104.

6. Nelson-Piercy C. Asthma in pregnancy. Thorax. 2001; 56:325-8.

7. Lee S, Kirking DM, Erickson SR. Methods of measuring asthma severity and influence on patient assignment. Ann Allergy Asthma Immunol. 2003; 91:449-54.

8. Rebuck DA, Hanania NA, D'Urzo AD, Chapman KR. The accuracy of a handheld portable spirometer. Chest. 1996; 109:152-7.

9. National Asthma Education Program. Report of the working group on asthma in pregnancy. Management of asthma during pregnancy. Bethesda: Department of Health and Human Services; 1993. (NIH publ. n. 93-3279).

10.ACOG technical bulletin. Pulmonary disease in pregnancy. Number 224-June 1996. American College of Obstetricians and Gynecologists. Int $\mathrm{J}$ Gynaecol Obstet. 1996; 54:187-96.

11.Lung function testing: selection of reference values and interpretation. American Thoracic Society. Am Rev Respir Dis. 1991; 144:1202-18.

12. Tabachnick BG, Fidell LS. Using multivariate statistics. $4^{\text {th }}$ ed. Boston: Allyn and Bacon; 1996. p. 31-5. 
13.Brancazio LR, Laifer SA, Schwartz T. Peak expiratory flow rate in normal pregnancy. Obstet Gynecol. 1997; 89:383-6.

14.Barreiro TJ, Perillo I. An approach to interpreting spirometry. Am Fam Physician. 2004; 69:1107-14.

15.Enright PL, Beck KC, Sherrill DL. Repeatability of spirometry in 18,000 adult patients. Am J Respir Crit Care Med. 2004; 169:235-8.

16.Jones KP, Mullee MA. Measuring peak flow in expiratory flow in general practice: comparison of mini Wright peak flow meter and turbine spirometer. BMJ. 1990; 300:1629-31.

17.Camargos PA, Ruchkys VC, Dias RM, Sakurai E. Accuracy of MiniWright peak flow expiratory flow meters. J Pediatr. 2000; 76:447-52.

18.De Swiet M. Pulmonary disorders. In: Creasy RK, Resnik R, editors. Maternal-fetal Medicine: principles and practice. $3^{\text {rd }}$ ed. Philadelphia: W.B. Saunders; 1994. p. 891-904.

19.Singh S, Singh KC, Sircar SS, Sharma KN. Airway functions in pregnant Indian women. Indian $\mathrm{J}$ Physiol Pharmacol. 1995; 39:160-2.

20.Puranik BM, Kurhade GA, Kaore SB, Patwardhan SA, Kher JR. PEFR in pregnancy: a longitudinal study. Indian J Physiol Pharmacol. 1995; 39:135-9.
21.Young SY, Gunzenhauser JD, Malone KE, McTiernan A. Body mass index and asthma in the military population of the northwestern United States. Arch Intern Med. 2001; 161:1605-11.

22.Tang RB, Chao T, Chen SJ, Lai CC. Pulmonary function during exercise in obese children. Zhongua Yi Xue Za Zhi (Taipei.) 2001; 64:403-7.

23.Pereira CAC, Barreto SP, Simões JG, Pereira FWL, Gerstler JG, Nakatani J. Valores de referência para a espirometria em uma amostra da população brasileira adulta. J Pneumol. 1992; 18:10-22.

24.Mauad-Filho F, Dias CC, Ramos D, Nogueira AA, Berezowski AT, Duarte D. Asma e gravidez: tratamento hospitalar. Rev Bras Ginecol Obstet. 2001; 23:523-7.

25.Schatz M, Zeiger RS, Hoffman CP, et al. Perinatal outcomes in the pregnancies of the asthmatic women: a prospective controlled analysis. Am J Respir Crit Care Med. 1995; 151:1170-4.

26.Demissie K, Breckenridge MB, Rhoads GG. Infant and maternal outcomes in the pregnancies of asthmatic women. Am J Respir Crit Care Med. 1998; 158:1091-5. 